\title{
BIFURCATION OF STEADY-STATE SOLUTIONS OF A SCALAR REACTION-DIFFUSION EQUATION IN ONE SPACE VARIABLE
}

\author{
SHIN-HWA WANG and NICHOLAS D. KAZARINOFF ${ }^{\dagger}$
}

(Received 3 January 1990; revised 7 September 1990)

Communicated by E. N. Dancer

\begin{abstract}
We study the bifurcation of steady-state solutions of a scalar reaction-diffusion equation in one space variable by modifying a "time map" technique introduced by J. Smoller and A. Wasserman. We count the exact number of steady-state solutions which are totally ordered in an order interval. We are then able to find their Conley indices and thus determine their stabilities.
\end{abstract}

1991 Mathematics subject classification (Amer. Math. Soc.): 58 F 14, 34 B 15.

Keywords and phrases: bifurcation, steady-state solutions, nonnegative solutions, two-point boundary value problem, stability, and Conley index.

\section{Introduction}

We study the bifurcation of steady-state solutions of a scalar reaction-diffusion equation in one space variable

$$
u_{t}-u_{x x}-f(u)=0, \quad(x, t) \in \Omega \times \mathbb{R}_{+} \subset \mathbb{R} \times \mathbb{R}_{+}
$$

together with the boundary conditions

$$
u(x, t)=0, \quad(x, t) \in \partial \Omega \times \mathbb{R}_{+}
$$

and initial data $u(x, 0)$. For proper choices of $f$, equation (1.1) models some chemical and biological diffusion phenomena $[2,4,10]$.

${ }^{\dagger}$ Deceased. Nicholas D. Kazarinoff died in November 1991. He was a fine mathematician and a respected friend.

(C) 1992 Australian Mathematical Society 0263-6115/92 \$A2.00+0.00 
In this paper, we shall concern ourselves with bounded spatial regions $\Omega=\{|x|<L\}$; this requires that $u$ satisfy bounded boundary conditions at $\pm L$. Then the steady-state equation associated with (1.1) and $\left(1.1^{\prime}\right)$ is the two-point boundary value problem

$$
u^{\prime \prime}+f(u)=0, \quad-L<x<L, \quad u(-L)=u(L)=0 .
$$

The real-valued function $f:[0, \infty) \rightarrow \mathbb{R}$ is initially assumed to be $C^{2}$ and to have exactly three nonnegative simple roots $0 \leq s_{0}<s_{1}<s_{2}$ with $f(0) \geq 0$. Furthermore, we also assume that the area of a "hill" exceeds that of the preceding "valley".

We obtain the local bifurcation diagram of positive solutions $u$ of (1.2) satisfying

$$
s_{0}<\|u\|_{\infty}<s_{2},
$$

for such a function $f$; that is, we count the exact number of nonnegative solutions in the order interval $\left[0, s_{2}\right)=\left\{u \mid 0 \leq u<s_{2}\right\}$. Notice that phaseplane analysis shows $f\left(\|u\|_{\infty}\right)>0$ if $u$ is a positive solution, and if $f(0)=$ 0 , then $u \equiv 0$ is always a steady-state solution (that is, for all $L$ ). We are interested in nonconstant positive solutions other than the trivial solution $u \equiv 0$, if it exists.

We study (1.2) through an approach due to J. Smoller and A. Wasserman [11] who studied (1.2) by the technique of "time map" $T(\alpha)$ to count the exact number of solutions of (1.2) for $f$ a cubic polynomial. Thus our method of proof is not new. We show that, for large $L$, if $f$ satisfies (2.1)-(2.3), then (1.2) has exactly three totally ordered positive solutions in the interval $\left(0, s_{2}\right)$ if $f(0)>0$ and (1.2) has exactly three totally ordered positive solutions in $\left(0, s_{2}\right)$ other than the trivial solution $u \equiv 0$ if $f(0)=0$ and $f^{\prime}(0)<0$.

Note. Our method of proof allows us to relax the $C^{2}$-hypothesis on $f$; we only need $f$ to be $C^{1}$.

The research in this paper is motivated by papers $[3,5]$ in which a multiplicity result of at least three totally ordered positive solutions of the Dirichlet problem

$\Delta u+\lambda f(u)=0$ in $\Omega\left(\Omega\right.$ is a smooth bounded domain in $\left.\mathbb{R}^{k}(k \geq 1)\right)$,

$$
u=0 \text { on } \partial \Omega \text {, }
$$

in the ordered interval $\left(0, s_{2}\right)$ was obtained separately by variational and topological index argument for function $f \in C^{1}$ such that $0<s_{0}<s_{1}<s_{2}$ and satisfying

(f1) $f(y)>0$ on $\left(0, s_{0}\right)$, or $\left(f 1^{\prime}\right) \quad f(0)=0$ and $f_{+}^{\prime}(0)>0$,

(f2) $f\left(s_{0}\right)=f\left(s_{1}\right)=f\left(s_{2}\right)=0$, 
(f3) $\int_{s_{0}}^{s_{2}} f(s) d s>0$

if $\lambda$ is large enough. More precisely, it was shown in $[3,5]$ that $(1.2)$ has at least one positive solution satisfying $0<\|u\|_{\infty}<s_{0}$ and at least two positive solutions satisfying (1.3) if $\lambda$ is large enough. Moreover, these three positive solutions obtained are totally ordered (see also [7]).

Note 1. Conditions (f2) and (f3) correspond to our assumptions (2.1) and part of (2.3). However, ( $\mathrm{fl}$ ) and ( $\left.\mathrm{fl}^{\prime}\right)$ are different from our assumption in the case that $f(0)=0$ where we assume $f_{+}^{\prime}(0)<0$ and where Dancer [5] assumed $f_{+}^{\prime}(0) \geq 0$ and where de Figueiredo [3] assumed $f_{+}^{\prime}(0)>0$.

Note 2. If we make change of variable $y=x / L$, then (1.2) becomes

$$
u_{y y}+L^{2} f(u)=0, \quad|y|<1, \quad u( \pm 1)=0,
$$

so that if $\lambda=L^{2}$, we obtain a problem of the type (1.4). We prefer, however, to consider the equation (1.2) because, as we shall see, its solutions can be given a nice geometrical interpretation [10, p. 185].

REMARK. A cubic polynomial $f$ cannot satisfy the conditions (2.1)-(2.3) of Theorem 1 stated in Section 2. Nevertheless, we should remark that for $f=-\left(x-s_{0}\right)\left(x-s_{1}\right)\left(x-s_{2}\right)$ satisfying (f4) $0<s_{0}<s_{1}<s_{2}$ and (f5) $\int_{s_{0}}^{s_{2}} f(s) d s>0$, the problem of the complete bifurcation diagram of solutions of (1.2) is still open. Only partial results are known; see the first author's paper [13] for details.

As Smoller and Wasserman did in [11], we rewrite (1.2) as a first order system

$$
u^{\prime}=v, \quad v^{\prime}=-f(u), \quad|x|<L,
$$

and we consider the phase plane for (1.6) locally illustrated in Figure 1 for $s_{0}>0$.

It is clear that positive solutions of (1.2) satisfying (1.3) correspond to those orbits of (1.6) which "begin" on the interval $\left(A_{0}, A_{1}\right) \quad\left(A_{0}>0, A_{1}>0\right.$ with $A_{0}^{2}=2 F\left(s_{0}\right)$ and $A_{1}^{2}=2 F\left(s_{2}\right)$, where $\left.F(s) \equiv \int_{0}^{s} f(u) d u\right)$ on the $v$ axis, and "end" on the $v$-axis, and take "time" (parameter length) $2 L$ to make the journey [11]. Then, as in [11], we use the time map

$$
T(\alpha)=2^{-1 / 2} \int_{0}^{\alpha}(F(\alpha)-F(u))^{-1 / 2} d u, \quad \gamma<\alpha<s_{2},
$$

where $\gamma \in\left(s_{1}, s_{2}\right)$ with $\int_{s_{0}}^{\gamma} f(s) d s=0$. Notice that solutions of (1.6) correspond to curves for which $T(\alpha)=L$. This led us to investigate the shape of graph of $T$; see [10, pp. 186-187]. We write as (1.8) and (1.9) below two formulas from [11]:

$$
T^{\prime}(\alpha)=2^{-3 / 2} \int_{0}^{\alpha} \frac{\theta(\alpha)-\theta(u)}{(\Delta F)^{3 / 2}} \frac{d u}{\alpha}
$$




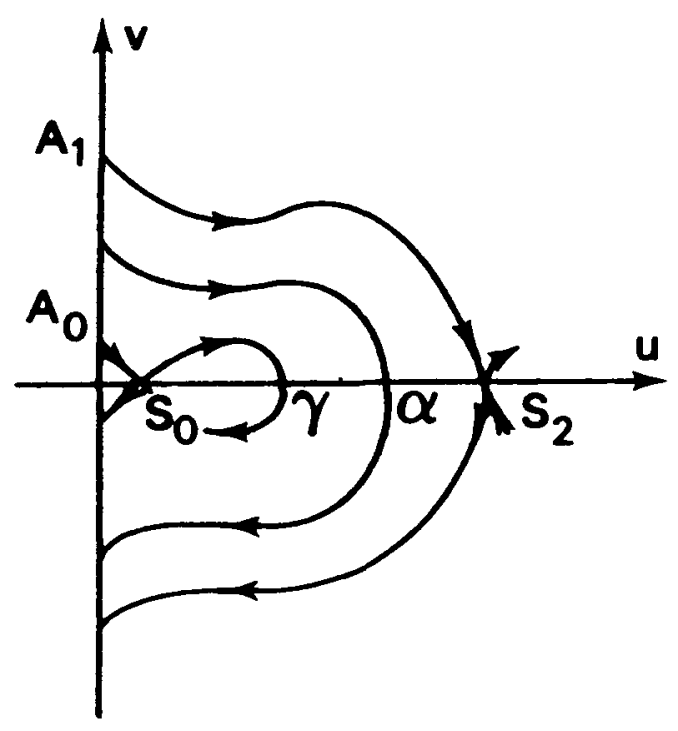

FIGURE 1

where $\Delta F=F(\alpha)-F(u)$ and $\theta(x)=2 F(x)-x f(x)$;

$$
T^{\prime \prime}(\alpha)+\frac{2}{\alpha} T^{\prime}(\alpha)>\frac{2^{-3 / 2}}{\alpha^{2}} \int_{0}^{\alpha}(\Delta F)^{-3 / 2}(\phi(\alpha)-\phi(u)) d u
$$

where $\phi(x)=x \theta^{\prime}(x)-\theta(x)$.

We analyze the "time map" $T$ by studying the convexity of the curve $y=f(x)$. We recall that the domain of $T$ is the open interval $\left(\gamma, s_{2}\right)$. Furthermore, from phase-plane analysis, we know that if $\alpha$ is near $\gamma$ or $s_{2}, T(\alpha)$ must be very large. Since $T$ is a smooth function, we see that $T(\alpha)$ must have at least one critical point, a minimum on $\left(\gamma, s_{2}\right)$, say at $\alpha_{0}$. Obviously, $T\left(\alpha_{0}\right)>0[10]$.

\section{Main Result}

THEOREM 1. Suppose $f \in C^{2}$, and there are numbers $0 \leq s_{0}<s_{1}<s_{2}$ such that the following conditions are satisfied:

$$
\begin{gathered}
f\left(s_{0}\right)=f\left(s_{1}\right)=f\left(s_{2}\right)=0 \\
f^{\prime \prime}(x)>0 \text { for } x \in\left(0, s_{1}\right), \quad f^{\prime \prime}(x)<0 \text { for } x \in\left(s_{1}, s_{2}\right)
\end{gathered}
$$


$\int_{s_{0}}^{s_{2}} f(s) d s>0$, and there exists $\gamma$ in $\left(s_{1}, s_{2}\right)$ defined by $\int_{s_{0}}^{\gamma} f(s) d s=0$

and such that $2 F(\gamma)-\gamma f(\gamma)<0$.

Let $T$ be defined by (1.7). Then $T$ has exactly one critical point, a minimum in $\left(\gamma, s_{2}\right)$.

EXAMPLE of Functions $f$. Choose

$$
f(x)= \begin{cases}-x(x-1)(x-2), & 0 \leq x \leq 1, \\ -\frac{1}{4}(x-1)^{3}+x-1, & 1<x \leq 3,\end{cases}
$$

where $s_{0}=0, s_{1}=1, s_{2}=3$, and $\gamma=1+\sqrt{2}$, or for $0<\varepsilon \ll 1$ sufficiently small, choose

$$
f(x)= \begin{cases}-(x-\varepsilon)(x-(1+\varepsilon))(x-(2+\varepsilon)), & 0 \leq x \leq 1+\varepsilon, \\ -\frac{1}{4}(x-(1+\varepsilon))^{3}+x-(1+\varepsilon), & 1+\varepsilon<x \leq 3+\varepsilon,\end{cases}
$$

where $s_{0}=\varepsilon, s_{1}=1+\varepsilon, s_{2}=3+\varepsilon$, and $\gamma=1+\sqrt{2}+\varepsilon$.

REMARK. No analysis of $f^{\prime \prime}$ was used in [11]; but it is of importance in our analysis; see also [14].

Note 1. Since $2 F(\gamma)-\gamma f(\gamma)=2 F\left(s_{0}\right)-\gamma f(\gamma)$, if $s_{0}=0$ in (2.3), then the condition $2 F(\gamma)-\gamma f(\gamma)=-\gamma f(\gamma)<0$ is automatically satisfied.

Nore 2. Conditions (2.1) and (2.2) imply (2.4) and (2.5) stated below.

$$
\begin{aligned}
f(x)>0 & \text { for } x \in\left[0, s_{0}\right) \quad \text { if } s_{0}>0, \\
f(x)<0 & \text { for } x \in\left(s_{0}, s_{1}\right), \\
f(x)>0 & \text { for } x \in\left(s_{1}, s_{2}\right) . \\
f^{\prime}\left(s_{0}\right)<0, & f^{\prime}\left(s_{1}\right)>0, \quad \text { and } \quad f^{\prime}\left(s_{2}\right)<0 .
\end{aligned}
$$

\section{PROOF OF THEOREM 1.}

Since we know that the "time map" $T$ has at least one critical point on $\left(\gamma, s_{2}\right)$, it suffices to show that $T$ has at most one critical point. For this we will show

$$
T^{\prime \prime}(\alpha)>0 \text { if } T^{\prime}(\alpha)=0 .
$$

Indeed, we shall show that $T^{\prime}(\alpha)<0$ for $\gamma<s \leq p_{2}$ (for $p_{2}$ defined below) and (2.6) holds on $\left(p_{2}, s_{2}\right)$ so that $T^{\prime}(\alpha)$ can vanish exactly once there (its zero lies on $\left.\left(p_{2}, s_{2}\right)\right)$. In (1.8), we have

$$
T^{\prime}(\alpha)=2^{-3 / 2} \int_{0}^{\alpha} \frac{\theta(\alpha)-\theta(u)}{(\Delta F)^{3 / 2}} \frac{d u}{\alpha}
$$


where $\theta(x)=2 F(x)-x f(x)$, which gives

$$
\theta^{\prime}(x)=f(x)-x f^{\prime}(x)
$$

and

$$
\theta^{\prime \prime}(x)=-x f^{\prime \prime}(x) \begin{cases}<0 & \text { if } x \in\left(0, s_{1}\right), \\ >0 & \text { if } x \in\left(s_{1}, s_{2}\right) .\end{cases}
$$

Now by (2.1), (2.3), and (2.4),

$$
\begin{aligned}
& \theta(0)=0, \\
& \theta\left(s_{0}\right)=2 F\left(s_{0}\right)=0 \text { if } s_{0}=0 ; \quad \theta\left(s_{0}\right)=2 F\left(s_{0}\right)>0 \text { if } s_{0}>0, \\
& \theta(\gamma)<0, \text { and } \\
& \theta\left(s_{2}\right)=2 F\left(s_{2}\right)>0 .
\end{aligned}
$$

So (i) if $s_{0}>0, \theta$ has one root at zero and at least two distinct positive roots, $q_{1}$ and $q_{2}$ with $s_{0}<q_{1}<\gamma<q_{2}<s_{2}$; and (ii) if $s_{0}=0$, then $\theta$ has one root at zero and at least one positive root, $q_{2}$, with $\gamma<q_{2}<s_{2}$. Also by (2.5) and (2.8), we know

$$
\begin{aligned}
& \theta^{\prime}(0)=f(0)>0 \text { if } s_{0}>0, \\
& \theta^{\prime}\left(s_{0}\right)=-s_{0} f^{\prime}\left(s_{0}\right)=0 \text { if } s_{0}=0 ; \quad \theta^{\prime}\left(s_{0}\right)=-s_{0} f^{\prime}\left(s_{0}\right)>0 \text { if } s_{0}>0, \\
& \theta^{\prime}\left(s_{1}\right)=-s_{1} f^{\prime}\left(s_{1}\right)<0, \text { and } \\
& \theta^{\prime}\left(s_{2}\right)=-s_{2} f^{\prime}\left(s_{2}\right)>0 .
\end{aligned}
$$

So by (2.9), (i) if $s_{0}>0$, then $\theta^{\prime}$ has exactly two positive roots, $p_{1}$ and $p_{2}$ with $s_{0}<p_{1}<s_{1}<p_{2}<s_{2}$; and (ii) if $s_{0}=0$, then $\theta^{\prime}$ has one zero root and exactly one positive root, $p_{2}$ with $s_{1}<p_{2}<s_{2}$. By the previous argument, (i) if $s_{0}>0$, then $\theta$ has exactly two distinct positive roots, $q_{1}$ and $q_{2}$ with $s_{0}<q_{1}<\gamma<q_{2}<s_{2}$; and (ii) if $s_{0}=0$, then $\theta$ has exactly one positive root, $q_{2}$, with $\gamma<q_{2}<s_{2}$. Note that

$$
\theta^{\prime}\left(p_{2}\right)=0 \text { and } \theta\left(p_{2}\right)<0,
$$

which we use to show (2.6).

One sees that the graph of $\theta$ is as in Figure 2 for $s_{0}>0$. Thus

$$
\theta(\alpha)-\theta(u)<0 \text { if } \gamma<\alpha<p_{2} \text { and } u<\alpha .
$$

So

$$
T^{\prime}(\alpha)<0 \text { if } \gamma<\alpha \leq p_{2} .
$$

Hence, to show (2.6), we need only to consider $\alpha>p_{2}$. 


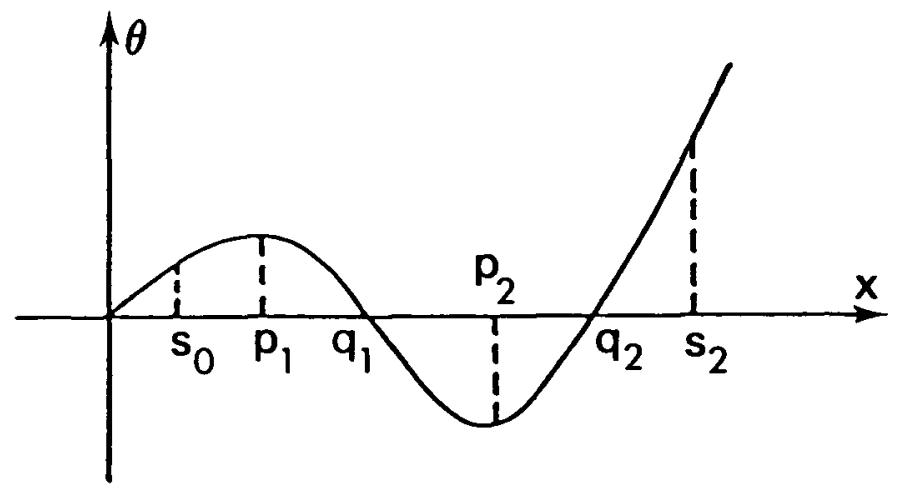

FIGURE 2

Now, from (1.9), we find that

$$
T^{\prime \prime}(\alpha)+\frac{2}{\alpha} T^{\prime}(\alpha)>\frac{2^{-3 / 2}}{\alpha^{2}} \int_{0}^{\alpha}(\Delta F)^{-(3 / 2)}(\phi(\alpha)-\phi(u)) d u,
$$

in which

$$
\phi(x)=x \theta^{\prime}(x)-\theta(x)
$$

So by (2.9)

$$
\phi^{\prime}(x)=x \theta^{\prime \prime}(x)=-x^{2} f^{\prime \prime}(x) \begin{cases}<0 & \text { if } x \in\left(0, s_{1}\right), \\ >0 & \text { if } x \in\left(s_{1}, s_{2}\right)\end{cases}
$$

It is easy to see that by (2.12) and (2.16),

$$
\phi(0)=0, \quad \phi\left(p_{2}\right)=p_{2} \theta^{\prime}\left(p_{2}\right)-\theta\left(p_{2}\right)=-\theta\left(p_{2}\right)>0 .
$$

Thus, we obtain the graph of $\phi$, given in Figure 3 for $s_{0}>0$; note that $s_{1}<p_{2}<s_{2}$.

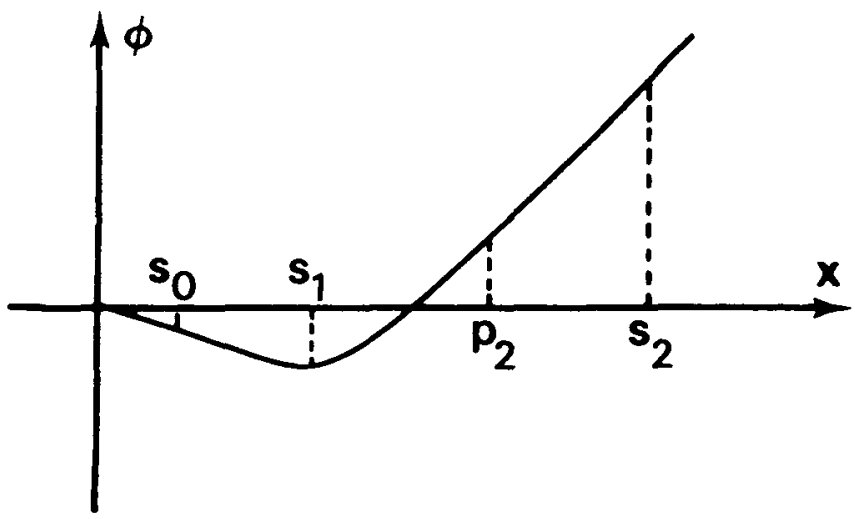

Figure 3 


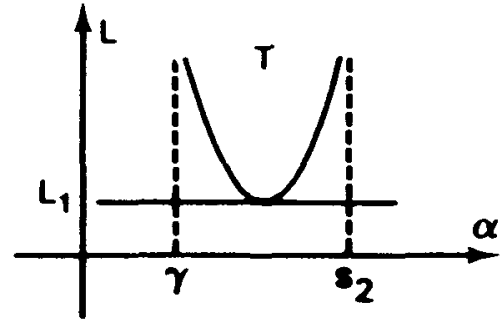

(a) $s_{0}=0$

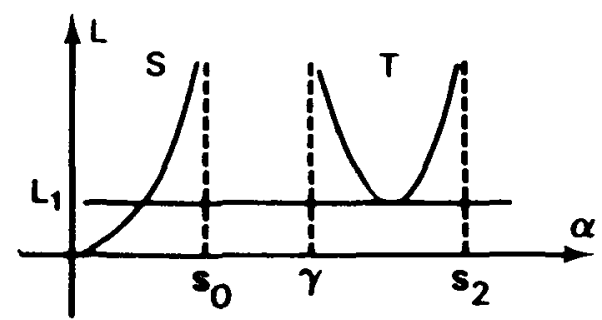

(b) $s_{0}>0$

Figure 4

We conclude that in the integrand of (2.15),

$$
\phi(\alpha)-\phi(u)>0 \text { if } p_{2}<\alpha<s_{2} \text { and } u<\alpha .
$$

Thus

$$
T^{\prime \prime}(\alpha)+\frac{2}{\alpha} T^{\prime}(\alpha)>0 \text { for } p_{2}<\alpha<s_{2},
$$

and if $T^{\prime}(\alpha)=0$ for some $\alpha, p_{2}<\alpha<s_{2}$, then $T^{\prime \prime}(\alpha)>0$. This and (2.14) imply (2.6). So $T^{\prime}$ vanishes at most once on $\left(\gamma, s_{2}\right)$. Hence $T$ has exactly one critical point, a minimum on $\left(\gamma, s_{2}\right)$. This completes the proof of Theorem 1.

REMARK 1. If one reviews the proof, one sees that the requirements of the smoothness and convexity conditions on function $f$ in (2.2) can be weakened; we can replace $f \in C^{2}$ by $f \in C^{1}$, and weaken (2.2) by requiring that $\theta$ and $\phi$ satisfy

(2.21) $\theta^{\prime}(x)=f(x)-x f^{\prime}(x)$ is strictly decreasing in $\left(0, s_{1}\right)$ and strictly increasing in $\left(s_{1}, s_{2}\right)$, and

(2.22) $\phi(x)=-2 F(x)+2 x f(x)-x^{2} f^{\prime}(x)$ is strictly decreasing in $\left(0, s_{1}\right)$ and strictly increasing in $\left(s_{1}, s_{2}\right)$.

RemarK 2. Condition (2.2) can be weakened as $f^{\prime \prime}>0$ in $(0, d), f^{\prime \prime}<$ 0 in $\left(d, s_{2}\right)$ for $d \in\left(c_{1}, c_{2}\right)$, where $c_{1}$ is the critical point of $f$ in $\left(s_{0}, s_{1}\right)$ and $c_{2}$ is the critical point of $f$ in $\left(s_{1}, s_{2}\right)$.

REMARK 3. If $s_{0}>0$, then the solution with $\|u\|_{\infty} \in\left(0, s_{0}\right)$ cannot undergo bifurcation. To see this we consider $T(\alpha)$ defined by (1.9) on $\left(\gamma, s_{2}\right)$ and on $\left(0, s_{0}\right)$, we define the "time map" $S(\alpha)$ by

$$
S(\alpha)=2^{-1 / 2} \int_{0}^{\alpha}(F(\alpha)-F(u))^{-1 / 2} d u, \quad 0<\alpha<s_{0} .
$$

By (2.1) and (2.2), $f(0)>0, f\left(s_{0}\right)=0$ and $f$ is strictly decreasing in 
$\left(0, s_{0}\right)$. It is easy to see that $S\left(0^{+}\right)=0, S\left(s_{0}^{-}\right)=+\infty$ and $S$ is strictly increasing in $\left(0, s_{0}\right)$; see also [7]. Combining the solution branches of $S$ and $T$, we see that the bifurcation diagram of (1.2) takes the form in Figure 4(a), (b). Therefore, for $L>L_{1}$, there are exactly three positive solutions if $s_{0}>0$ and exactly two positive solutions if $s_{0}=0$ in the order interval $\left(0, s_{2}\right)$ for $(1.2)$ if the function $f$ satisfies $(2.1)-(2.3)$.

\section{One Generalization}

Suppose $f$ has $2 m+1 \quad(m \geq 2)$ nonnegative simple roots $0 \leq s_{0}<s_{1}<$ $s_{2}<\cdots<s_{2 m-1}<s_{2 m}$ and also assume that the area of a "hill" exceeds that of the preceding "valley". Similarly to (1.7), for $n=1,2, \ldots, m$, we define $A_{n}>0$ by $A_{n}^{2}=2 F\left(s_{2 n}\right)$, and the "time map"

$$
T_{n}(\alpha)=2^{-1 / 2} \int_{0}^{\alpha}(F(\alpha)-F(u))^{-1 / 2} d u, \quad \gamma_{n}<\alpha<s_{2 n},
$$

where $\gamma_{n} \in\left(s_{2 n-1}, s_{2 n}\right)$ with $\int_{s_{2 n-2}}^{\gamma_{n}} f(s) d s=0$. As before, solutions of (1.6) correspond to curves for which $T_{n}(\alpha)=L$. Our argument in Section 2 can be easily modified to show that each "time map" $T_{n}$ has exactly one critical point for each $n=1,2, \ldots, m$. We now state without proof the following generalized theorem (recall that $\theta(x)=2 F(x)-x f(x)$ and $\phi(x)=$ $\left.x \theta^{\prime}(x)-\theta(x)=-2 F(x)+2 x f(x)-x^{2} f^{\prime}(x)\right)$.

Theorem 2. Suppose $f \in C^{2}$, and there are numbers $0 \leq s_{0}<s_{1}<s_{2}<$ $\cdots<s_{2 m-1}<s_{2 m}(m \geq 2)$ such that the following conditions are satisfied:

$$
f\left(s_{n}\right)=0 \text { for } n=0,1,2, \ldots, 2 m,
$$

$$
\begin{aligned}
& f^{\prime \prime}(x)>0 \text { for } x \in\left(0, s_{1}\right), \\
& f^{\prime \prime}(x)<0 \text { for } x \in\left(s_{2 n-1}, s_{2 n}\right), n=1,2, \ldots, m,
\end{aligned}
$$$$
f^{\prime \prime}(x)>0 \text { for } x \in\left(s_{2 n-2}, s_{2 n-1}\right), n=1,2, \ldots, m \text {, }
$$

$\int_{s_{2 n-2}}^{s_{2 n}} f(s) d s>0, \quad n=1,2, \ldots, m$, and there exists $a$ $\gamma_{n}$ in $\left(s_{2 n-1}, s_{2 n}\right)$ defined by $\int_{s_{2 n-2}}^{\gamma_{n}} f(s) d s=0$ and such that $\theta\left(\gamma_{n}\right)=2 F\left(\gamma_{n}\right)-\gamma_{n} f\left(\gamma_{n}\right)<0, n=1,2, \ldots, m$,

there exists $p_{2 n}$, a root of $f(x)-x f^{\prime}(x)=0$ in $\left(s_{2 n-1}, s_{2 n}\right)$, for $n=1,2, \ldots, m$ such that the following two conditions are satisfied: 


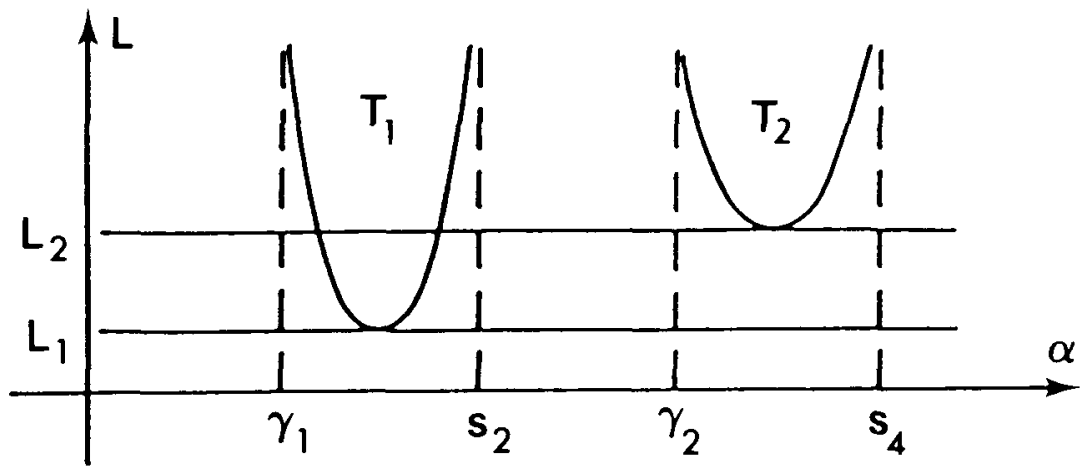

(a) $\mathrm{m}=2$ and $\mathrm{s}_{0}=0$

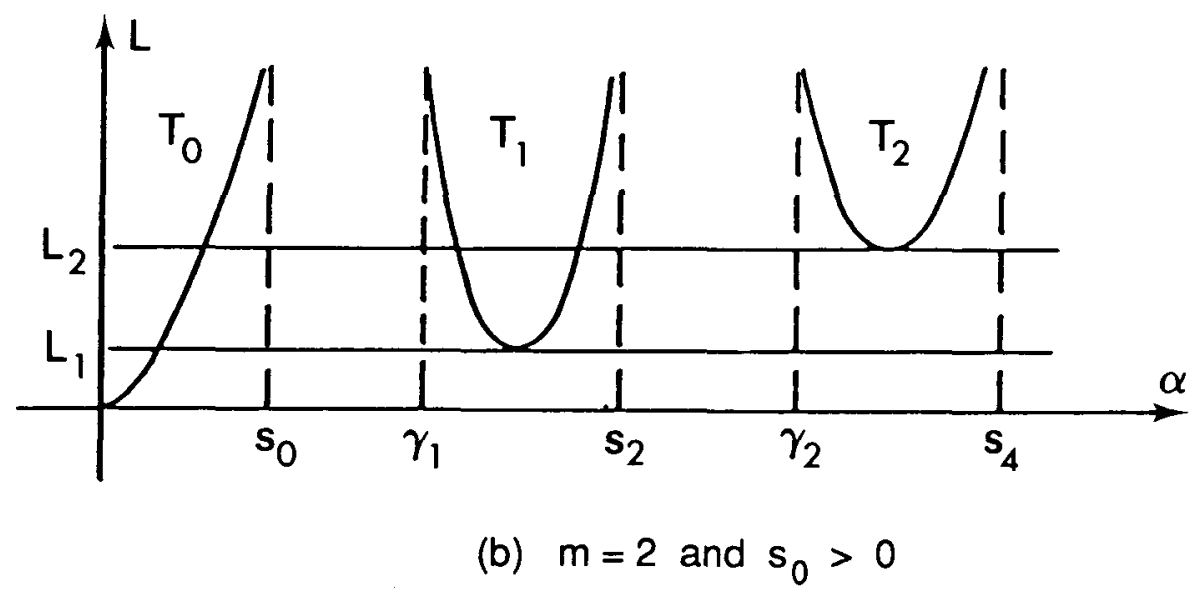

Figure 5

(1) either $p_{2 n} \leq \gamma_{n}$ for $n=2,3, \ldots, m$ or if $p_{2 n}>\gamma_{n}$ for $n=2,3, \ldots, m$ then $\theta\left(\gamma_{n}\right) \leq \theta\left(p_{2 n-2}\right)$

and

(2) $\phi\left(s_{2 n-2}\right) \leq \phi\left(p_{2 n}\right)$ for $n=2,3, \ldots, m$; i.e., $-2 F\left(s_{2 n-2}\right)-s_{2 n-2}^{2} f^{\prime}\left(s_{2 n-2}\right) \leq-2 F\left(p_{2 n}\right)+2 p_{2 n} f\left(p_{2 n}\right)$.

Let $T_{n}$ be defined by (3.1). Then for $n=1,2, \ldots, m, T_{n}$ has exactly one critical point, a minimum. 
REMARK 1. The proof of Theorem 2 is not very different from that of Theorem 1. Condition (3.5) is assumed to ensure the functions $\theta$ and $\phi$ have the desired properties and hence give the conclusion of Theorem 2.

REMARK 2. We can weaken the hypotheses on $f, \theta$, and $\phi$ in Theorem 2 as we did in (2.21) and (2.22) in Theorem 1.

REMARK 3. If $s_{0}>0$, on $\left(0, s_{0}\right)$ we define the "time map" $T_{0}$ to be the "time map" $S$ defined by (2.23). One sees that the bifurcation diagram of (1.2) if $f$ satisfies (3.2)-(3.5) takes the form given in Figure 5(a) for $m=2$ if $s_{0}=0$ in Figure $5\left(\right.$ b) for $m=2$ if $s_{0}>0$.

Thus for $0<L<\min _{n=1, \ldots, m}\left\{L_{n}\right\}$, there is only one nonnegative solution $u$, with $0 \leq\|u\|_{\infty}<s_{0}$. But for $L=L_{n} \quad(n=1,2, \ldots, m)$, a positive solution $u$ with $s_{2 n-1}<\|u\|_{\infty}<s_{2 n}$ appears. While for $L>L_{n} \quad(n=$ $1,2, \ldots, m)$, this solution bifurcates into two positive distinct solutions $u_{2 n-1}, u_{2 n}$ with $s_{2 n-1}<\left\|u_{2 n-1}\right\|_{\infty},\left\|u_{2 n}\right\|_{\infty}<s_{2 n}$. Therefore, for $L>$ $\max _{n=1, \ldots, m}\left\{L_{n}\right\}$, there are exactly $2 m+1$ positive solutions if $s_{0}>0$ and $2 m$ positive solutions other than the trivial solution $u \equiv 0$ if $s_{0}=0$ in the order interval $\left[0, s_{2 m}\right)$ for (1.2) if $f$ satisfies (3.2)-(3.5).

\section{A Remark on Total Ordering of Multiple Steady-States Solutions}

In this short section, we show the $2 m+1 \quad(m \geq 1)$ steady-state solutions $u_{0}, u_{1}, u_{2}, \ldots, u_{2 m-1}, u_{2 m}$ of (1.2) obtained in Theorems 1 and 2 for large $L$ are totally ordered. We have

THEOREM 3.

$$
u_{0}<u_{1}<u_{2}<\cdots<u_{2 m-1}<u_{2 m}
$$

Theorem 3 is an easy consequence of a special case of the following lemma, which can be shown by considering the first order system (1.6) and observing that the total energy function $H(u, v)=v^{2} / 2+F(u)$ is constant along orbits of (1.6). By $w<\hat{w}$, we mean $w(x)<\hat{w}(x), x \in(-L, L)$.

LEMMA 1. Let $w$ and $\hat{w}$ be any two distinct positive solutions of (1.2) with $0<\|w\|_{\infty}<\|\hat{w}\|_{\infty}$. Then

$$
w<\hat{w}
$$

Note 1. Lemma 1 says any two distinct positive solutions of (1.2) are ordered.

Note 2. In Lemma $1, f$ is not necessary to satisfy (3.2) and the first part of (3.4). We do not require $L$ to be large enough (cf. [3]).

Note 3. $\|w\|_{\infty}=w(0)$ and $\|\hat{w}\|_{\infty}=\hat{w}(0)$. It is easy to see that $\|w\|_{\infty} \neq$ 
$\|\hat{w}\|_{\infty}$ by the existence and uniqueness theorem for autonomous system [8, p. 162].

\section{A Brief Remark on Stability of the Multiple Steady-State Solutions}

In this section we briefly discuss the stability of these $2 m+1 \quad(m \geq 1)$ steady-state solutions

$$
u_{0}, u_{1}, u_{2}, \ldots, u_{2 m-1}, u_{2 m}
$$

obtained in Theorems 1,2 for $L>\max _{n=1, \ldots, m}\left\{L_{n}\right\}$ by a powerful topological tool, the Conley index theory. We get more information about the global structure of the multiple steady-states. Much of the exposition given here is adapted from Smoller [11]. It can be shown that the Conley index of $u_{2 n-1}$, $h\left(u_{2 n-1}\right)=\Sigma^{1}$, a pointed one-sphere $(n=1,2, \ldots, m)$, and the Conley index of $u_{2 n}, h\left(u_{2 n}\right)=\Sigma^{0}$, a pointed zero-sphere $(n=1,2, \ldots, m)$. Then there exist solutions $v_{2 n-1}$ and $v_{2 n}$ of (1.1), which connect $u_{2 n-1}$ to $u_{2 n-2}$ and $u_{2 n-1}$ to $u_{2 n}(n=1,2, \ldots, m)$ respectively; that is,

$$
\begin{aligned}
& \lim _{t \rightarrow-\infty} v_{2 n-1}(x, t)=u_{2 n-1}(x), \quad \lim _{t \rightarrow+\infty} v_{2 n-1}(x, t)=u_{2 n-2}(x), \\
& \lim _{t \rightarrow-\infty} v_{2 n}(x, t)=u_{2 n-1}(x), \quad \lim _{t \rightarrow+\infty} v_{2 n}(x, t)=u_{2 n}(x),
\end{aligned}
$$

uniformly for $|x| \leq L$.

We now state results about the stability of the steady-state solutions of (1.1) and $\left(1.1^{\prime}\right)$.

Proposition 1. Let $f$ satisfy (3.2)-(3.5), and $L>\max _{n=1, \ldots, m}\left\{L_{n}\right\}$. Then there are exactly $2 m+1 \quad(m \geq 1)$ steady state solutions, $u_{n} \quad(n=$ $0,1,2, \ldots, 2 m)$ of $(1.1)$ and $\left(1.1^{\prime}\right)$ with $0 \leq\left\|u_{0}\right\|_{\infty}<s_{0}, s_{2 n-2}<$ $\left\|u_{2 n-1}\right\|_{\infty},\left\|u_{2 n}\right\|_{\infty}<s_{2 n} \quad(n=1,2, \ldots, m)$. Each solution $u_{2 n}$ is stable and each $u_{2 n-1}$ has a one-dimensional unstable manifold which consists of orbits connecting $u_{2 n-1}$ to $u_{2 n-2}$ and $u_{2 n}$. All solutions of the problem are depicted (qualitatively) in Figure 6 . Initial data $u(x, 0)$, which satisfies the condition $u_{2 n-1}(x)<u(x, 0)<u_{2 n}(x)$ (respectively $u_{2 n-2}(x)<u(x, 0)<$ $\left.u_{2 n-1}(x)\right)$ on $|x|<L$ is in the stable manifold of $u_{2 n}$ (respectively $u_{2 n-2}$ ) $(n=1,2, \ldots, m)$. 


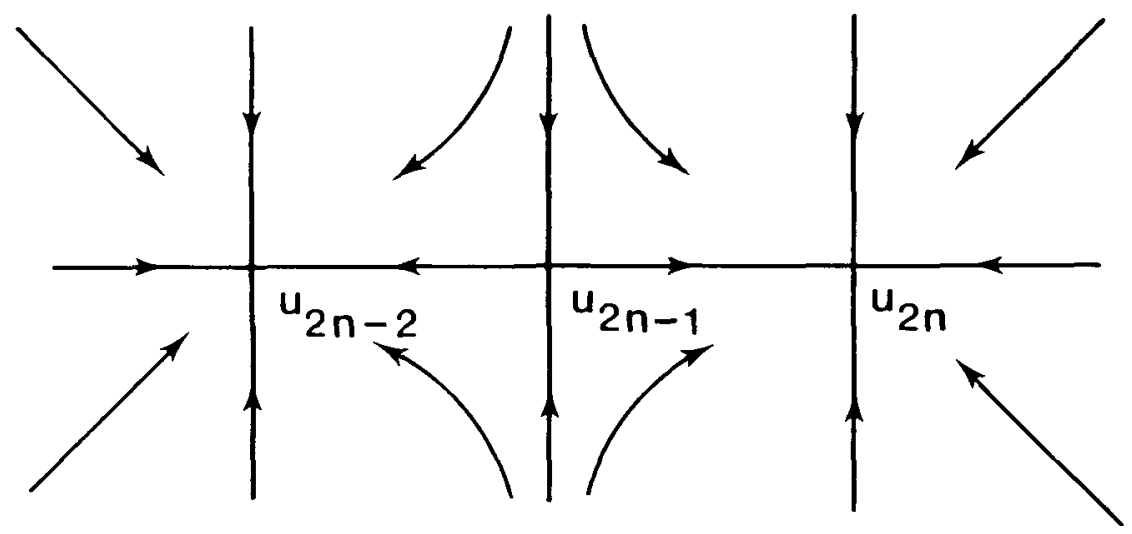

Figure 6

\section{References}

[1] K. J. Brown and H. Budin, 'On the existence of positive solutions for a class of semilinear elliptic boundary value problems', SIAM J. Math. Anal. 10 (1979), 875-883.

[2] C. Conley and J. Smoller, 'Bifurcation and stability of stationary solutions of the FitzHugh-Nagumo equations', J. Differential Equations 63 (1986), 389-405.

[3] E. N. Dancer, 'Multiple fixed points of positive mappings', J. Reine Angew. Math. 352 (1986), 46-66.

[4] P. C. Fife, Mathematical Aspects of Reacting and Diffusing Systems, (Springer-Verlag, Berlin, Heidelberg, New York, 1979).

[5] D. G. de Figueiredo, 'On the existence of multiple ordered solutions of nonlinear eigenvalue problems', Nonlinear Anal. 11 (1987), 481-492.

[6] B. Gidas, W. M. Ni, and L. Nirenberg, 'Symmetry and related properties via the maximum principle', Comm. Math. Phys. 68 (1979), 209-243.

[7] P. Hess, 'On multiple positive solutions of nonlinear elliptic equations', Comm. Partial Differential Equations 6 (1981), 951-961.

[8] M. Hirsch and S. Smale, Differential Equations, Dynamical Systems, and Linear Algebra, (Academic Press, New York, 1974).

[9] T. I. Seidman, 'Asymptotic growth of solutions of $-\Delta u=\lambda f(u)$ for large $\lambda$ ', Indiana Univ. Math. Journal 30 (1981), 305-311.

[10] J. Smoller, Shock Waves and Reaction-Diffusion Equations, (Springer-Verlag, New York, 1983).

[11] J. Smoller and A. Wasserman, 'Global bifurcation of steady-state solutions', J. Differential Equations 39 (1981), 269-290.

[12] J. Smoller and A. Wasserman, 'Generic bifurcation of steady-state solutions', J. Differential Equations 52 (1984), 432-438.

[13] S.-H. Wang, 'A correction for a paper by J. Smoller and A. Wasserman', J. Differential Equations 77 (1989), 199-202.

[14] S.-H. Wang and N. D. Kazarinoff, 'Bifurcation and Stability of Positive Solutions of a Two-Point Boundary Value Problem', J. Austral. Math. Soc. (Series A) 52 (1992), 334-342.

Department of Mathematics

National Tsing Hua University

Hsinchu, Taiwan 300, R.O.C. 